\title{
Quand construire une position émotionnelle, c'est choisir une conclusion argumentative : le cas d'un café-débat sur l'eau potable au Mexique ${ }^{1}$
}

Le rôle des émotions dans le fonctionnement des discours argumentatifs a été longtemps sous-étudié. Il n'a été réhabilité en tant qu'objet d'étude légitime que récemment, dans une perspective radicalement descriptive, fondée sur l'analyse d'interactions authentiques. Dans un tout autre champ, la reconnaissance de l'importance de traiter en classe de ce que la didactique anglo-saxonne appelle les «questions socio-scientifiques 》 (Albe 2006 ; Fowler, Zeidler, \& Sadler 2009 ; Kacem \& Simonneaux 2009 ; Nuangchalerm 2010) brouille les distinctions traditionnelles entre sciences naturelles et sciences sociales, subjectivité et objectivité, connaissance et opinion, et offre un espace a priori propice à la mobilisation des émotions à des fins argumentatives. Au travers de l'analyse d'un débat entre élèves sur l'accès à l'eau potable, cet article donne à voir que, dans l'argumentation ordinaire, la défense d'une conclusion argumentative est intrinsèquement liée à la construction discursive d'une position émotionnelle ${ }^{2}$. Celle-ci est donnée à voir par un travail de schématisation (Grize 1996) de la situationproblème ainsi que des différentes alternatives de réponse, qui produit une orientation émotionnelle, partie intégrante de l'orientation argumentative du discours. Nous cherchons à «suivre» ce travail de schématisation émotionnelle au travers de différentes traces discursives, à partir des outils développées par Plantin (2011) pour l'étude des émotions en argumentation.

1 Les auteurs remercient le concours que la région Rhône-Alpes a apporté à leur travail via l'attribution d'une allocation doctorale de recherche à Claire Polo.

2 Nous reconnaissons l'intérêt de la distinction de Caffi \& Janney (1994) entre "émotionnel» et « émotif», le premier faisant référence aux émotions ressenties par le locuteur, tandis que le second adjectif renvoie aux émotions que le locuteur donne à voir dans une situation de communication (plus ou moins explicitement). Nous nous intéressons ici plutôt à l'émotif, puisque nous étudions les émotions inscrites dans le discours, sans prétention à connaître les émotions effectivement éprouvées par les locuteurs. Cependant, pour des commodités de langage, nous employons plutôt le terme d'« émotionnel». 


\section{Ancrage théorique : la construction discursive des émotions en argumentation}

Alors que les émotions occupent une grande place dans la rhétorique ancienne, l'autonomisation du champ des études de l'argumentation s'est accompagnée d'une distinction entre d'une part le modèle de démonstration de la logique formelle et d'autre part la «persuasion ». En conséquence, les émotions sont les grandes absentes des travaux qui marquent la refondation moderne de la discipline, comme par exemple l'ouvrage de Toulmin (1958). Même le projet de Olbrechts-Tyteca et Perelman (1958), qui concilie subjectivité et rationalité, fait peu mention du rôle des émotions dans l'argumentation, et s'en tient aux "valeurs», auxquelles est transféré le potentiel dynamique des émotions «positives $»^{3}$. Plus récemment, dans la théorie critique de l'argumentation, les émotions n'ont de place qu'en tant qu'indices du caractère fallacieux (Hamblin, 1970), ou potentiellement raisonnable ou fallacieux (Walton 1992) des arguments. Cependant, des travaux récents, notamment inspirés de l'analyse de situations argumentatives authentiques, témoignent d'une forte présence des émotions dans l'argumentation, et ont conduit à une analyse plus complexe de leur(s) fonction(s) dans le discours argumentatif (e. g. Plantin, Doury \& Traverso 2000 ; Micheli 2010 ; Plantin 2011). Dans une perspective descriptive, l'enjeu n'est ni de juger de la validité des arguments, ni de décrire l'état émotionnel réel des personnes, mais de comprendre comment les interactants, en situation d'argumentation, mobilisent, dans leur discours, des émotions à des fins argumentatives. Toute la difficulté d'une telle analyse réside dans le fait que la manifestation des émotions peut prendre des formes diverses, et est souvent implicite, ne se limitant pas à l'emploi d'un lexique de l'émotion : "Un discours peut véhiculer une émotion même si les acteurs de ce discours n'en explicitent aucune » (Plantin 2011 : 132).

Plantin propose plusieurs critères pour étudier les émotions dans le discours, allant de l'analyse lexicale fine à la prise en compte d'inférences émotionnelles basées sur des stéréotypes culturels. L'analyse des émotions non explicitement thématisées recouvre un continuum comprenant des inférences supposant un plus ou moins grand degré d'implicite. Ainsi, la distinction faite par Micheli (2013) entre émotions «montrées» et « étayées» peut être comprise comme une différence quant à ce qui est inféré. L'émotion «montrée » est reconstituée grâce à des stéréotypes

3 En effet, le terme de «valeurs » est un substitut à celui, péjoratif, d' "émotions » : "notons que les passions, en tant qu'obstacles, ne doivent pas être confondues avec les passions qui servent d'appui à une argumentation positive, et qui seront d'habitude qualifiées à l'aide d'un terme moins péjoratif, tel que valeur par exemple » (Traité, p. 630 ; cité dans Plantin 2011 : $55)$. 
culturels portant sur l'expression de l'émotion, c'est-à-dire sur des signaux «aval», des symptômes habituellement associés au ressenti émotionnel, comme par exemple «avoir le rouge aux joues ». L'émotion «étayée» repose, elle, sur des inférences plus larges, fonctionnant à partir de signaux «amont», soit de stéréotypes portant sur les formats de situations susceptibles de produire une émotion donnée. Par exemple, dans la culture occidentale, on s'attend généralement à ce qu'un enterrement soit triste. Concernant le fonctionnement de ces mécanismes inférentiels, Plantin (2011) distingue deux axes structurants selon lesquels se construit l'émotion dans le discours : l'axe de l'agrément (plaisir-déplaisir) et l'axe de l'intensité (émotion forte - émotion faible). Plantin a identifié un certain nombre de présupposés culturels qui, employés dans le discours, le «cadrent» émotionnellement selon ces deux axes. Par exemple, schématiser une situation comme plus ou moins proche ou lointaine, contrôlable ou incontrôlable, relevant de la fatalité ou imputable à une cause ou un agent défini(e) produit des tonalités émotionnelles différentes, plus ou moins intenses. Sur le plan de l'agrément, tout ce qui a trait à la vie est généralement perçu positivement, tandis que ce qui a trait à la mort l'est négativement. Présenter quelque chose comme étant conforme ou nonconforme à des normes partagées conduit non seulement à un jugement plus ou moins positif ou négatif, mais également à une émotion plus ou moins agréable.

Nous nous proposons de décrire, dans le contexte de cette étude de cas, comment les élèves cadrent la question en débat, et positionnent émotionnellement les différentes alternatives de réponse selon ces paramètres, et comment, ce faisant, ils orientent leur discours vers une conclusion argumentative.

\section{Présentation du corpus}

\subsection{Informations ethnologiques et séquence pédagogique ${ }^{4}$}

Les données présentées ici ont été recueillies lors d'un débat organisé en novembre 2011 dans un collège-lycée mexicain privé situé dans l'état de Puebla. Il a été animé par des lycéens formés à cet effet, pour un public de collégiens (13-14 ans). L'évènement dure 110 minutes et est rythmé par un questionnaire à choix multiples alternant devinettes assorties d'informations de base sur le sujet et questions d'opinion, donnant lieu à un débat en petits groupes, puis en classe-entière. L'objectif est d'explorer la thématique pour pouvoir débattre de la question principale $(\mathrm{QP})$, posée dès le début:

4 La séquence pédagogique a été co-conçue dans un partenariat entre le laboratoire ICAR et l'Association Rhône-Alpes des Petits Débrouillards (http://www.lespetitsdebrouillards-ra.org/). 
A tu parecer, el acceso al agua potable para una persona, en el futuro, dependerá sobre todo de: a) su ingreso económico b) su resistencia fisica a vivir con agua de menor calidad c) los esfuerzos hechos ahora mismo para ahorrar agua y preservarla d) su lugar de origen en el planeta e) la capacidad de la naturaleza a adaptarse a nuestras necesidades de consumo de agua f) de los avances cientificos $^{5}$

En fin de débat, les élèves reviennent à cette question, et doivent choisir, par groupe de quatre, une réponse parmi les 6 items proposés. Ils discutent ensuite leur position tous ensemble, puis choisissent anonymement une option personnelle.

Le passage du débat étudié dans cet article correspond à l'étape finale de discussion en classe entière de la question principale. Il est caractérisé par un fort investissement des élèves, qui en viennent même parfois à transformer le cadre participatif, en se répondant directement sans passer par la médiation de l'animatrice. Enfin, ce moment du débat présente un cas rare de recours explicite à une émotion dans plusieurs interventions publiques adressées à l'ensemble de la classe.

\subsection{Dispositif d'enregistrement}

D'un point de vue technique, il s'agit de l'unique évènement de ce type si bien documenté. Ont été recueillis : des enregistrements audio-visuels d'une vue de l'ensemble de la classe ainsi que de quatre autres vues (chacune des tables des élèves disposés en petits groupes), et de l'écran vidéo-projeté ; des photographies des notes prises au tableau, ainsi que les résultats des votes individuels anonymes de chaque élève.

\section{Une analyse intégrée des émotions en argumentation : co- construction de la tonalité émotionnelle et de l'orientation argumentative}

\subsection{Positionnement thymique du débat}

Plantin (2011) reprend les catégories psychiques de thymique et phasique pour analyser la construction discursive des émotions à visée argumentative. Le thymique renvoie à un état émotionnel relativement stable, toile de fond où surviennent des évènements émotionnels de type phasique, de plus grand intensité, et de moindre durée. Ici, la tonalité

\footnotetext{
5 « Selon toi, de quoi dépendra le plus à l'avenir l'accès d'une personne à l'eau potable ? a) de son revenu économique, b) de sa capacité physique à vivre avec de l'eau de moindre qualité, c) des efforts faits dès maintenant pour économiser l'eau et la préserver, d) de l'endroit où elle naîtra sur la planète, e) de la capacité de la nature à s'adapter à nos besoins en eau, f) des avancées scientifiques ».
} 
thymique du débat est particulièrement "grave»: les participants construisent le problème comme très intense et très désagréable dès le début de la séquence, et sans que cela ne soit remis en question.

Des traces de ce cadrage émotionnel sont présentes tout au long du débat. Un mécanisme particulièrement visible constitue le positionnement $\mathrm{du}$ problème sur l'axe vie-mort. En effet, en termes d'agrément, il est communément admis, en référence à un préconstruit culturel, que la mort et ce qui l'avoisine s'accompagnent d'émotions négatives, alors que ce qui a trait à la vie est associé à des émotions positives (Plantin 2011). La tonalité grave du débat ne découle pas de la nature intrinsèque du problème, mais surtout de l'insistance des participants sur le risque sérieux d'un manque d'eau entraînant la mort.

\section{Une question de "vie » ou de " mort» : traces lexicales}

La gravité de la Question ${ }^{6}$ en jeu est construite au fil du discours des élèves, qui la traitent, littéralement, comme une question de vie ou de mort. C'est le cas d'Alejandro, qui, en choisissant la réponse B (qui met en avant le facteur de « résistance physique »), estime que les gens se battront pour l'eau, et que seuls les plus forts survivront ${ }^{7}$ :

8 ALE (...) la desesperación de la demás gente que no tiene agua la gente pobre yo creo que se van a estar peleando por el como por conseguirla $(\ldots)^{8}$

Mais aussi de Gaspar, pro-A :

25 GAS (...) el dinero en comprar el agua para vivir (...) mucha gente puede morir por la falt- la falta de agua

ou d'Emilia, pro-A, qui fait une analogie avec des situations de famine :

$6 \mathrm{Au}$ sens de Plantin, lorsqu'il définit une situation argumentative : «du point de vue du dialogue, peut être considéré comme argumentatif tout discours produit dans un contexte de débat orienté par une Question » (Plantin 1996 : 24).

7 Dans les éléments qui suivent, le premier chiffre correspond au numéro du tour de parole. Seuls les tours proprement verbaux sont numérotés. L'ensemble de l'extrait en compte 81, dont 31 interventions des animateurs, principalement pour distribuer la parole. On notera que les conventions de transcription sont celles du groupe ICOR, intégralement décrites à la section « conventions de transcription» de la page icar.univ-lyon2.fr/projets/corinte/. Deux exceptions dérogent à ces règles : l'emploi d'une majuscule pour les lettres correspondant à des réponses possibles, et du symbole “(.)” pour toutes les pauses, qui n'ont pas été mesurées.

8 « le désespoir des autres gens qui n'ont pas d'eau les gens pauvres je crois qu'ils vont se battre pour l- comment pour en obtenir $»$.

9 « l'argent pour acheter l'eau pour vivre (...) beaucoup de gens peuvent mourir par manqmanque d'eau » 
64 EMI (...) igual que: por decir los niños que están moriendo de hambre en Áfrical ${ }^{10}$

De plus, l'emploi du verbe «agotar(se)»((s')épuiser), introduit par Gaspar dans la toute première intervention de la discussion, et repris par la suite à deux reprises, montre bien comment le débat tend à s'ancrer du côté de ce risque de mort :

2 GAS se va a empezar a agotar bueno ya se está agotando el agua $^{11}$

21 EMI ya lo estamos agotando ${ }^{12}$

$60 \mathrm{EDU}$ (lo que ahorramos) se va a agotar ${ }^{13}$

En effet, voici la définition de "agotar" de la Real Academia de la lengua Española (2001) :

1. Extraer todo el líquido que hay en una capacidad cualquiera.

(Extraire tout le liquide qu'il y a dans une capacité quelconque).

2. Gastar del todo, consumir. Agotar el caudal, las provisiones (...).

(Utiliser entièrement, consommer. Épuiser le flux, les provisions

(...).)

3. Cansar extremadamente. (Fatiguer extrêmement).

Les sens 2 et 3 sont clairement orientés vers la mort, par l'épuisement extrême des forces. On peut également associer le sens 1 à une métaphore stéréotypique de la mort, avec le symbole de se vider de ses forces, de son eau, ou de son sang.

La nécessité : construction d'une chaîne causale débouchant sur le risque de mort

Tous les élèves ne font pas mention directe d'un lexique évoquant la mort. Beaucoup concourent à ce cadrage thymique de façon plus indirecte, en insistant sur le manque d'eau, réel ou potentiel. Il apparaît comme un leitmotiv, tout au long du débat, et transcende les clivages entre les différentes conclusions soutenues. Le manque est présenté comme un problème affectant une catégorie de population particulière, comme les pauvres :

$62 \mathrm{OAN}$ (...) los ricos tendrán el agua que quieran y los pobres nol $(\ldots)^{14}$

10 « comme euh: pour dire les enfants qui meurent de faim en Afrique »

11 « elle va commencer à s'épuiser bon l'eau est déjà en train de s'épuiser »

12 « nous sommes déjà en train de l'épuiser»

13 « (ce que nous économisons) va s'épuiser» 
$76 \operatorname{MAR}\left(\right.$... ni va a poder tomarla $\left.\right|^{15}$

Mais les formules employées par les élèves sont souvent plus radicales, et renvoient à une absence totale d'eau, que ce soit pour une catégorie de personnes :

$8 \mathrm{ALE}$ (...) la demás gente que no tiene agua (...) ${ }^{16}$

51 ART (...) tú no vas a tener (...) $)^{17}$

64 EMI (...) la gente que no tiene dinero se va a quedar sin agua tal vez a lo mejor en esos lugares donde se van a quedar sin agua (... $)^{18}$

ou même pour l'ensemble de la société :

4 EDU si no hay agua ahorrada cómo van a: este avance cientifico $\sin$ agua $^{19}$

Cette radicalisation participe de la gravité émotionnelle de la tension thymique de base, en rapprochant la situation décrite de la mort. Si cet enchaînement causal reste implicite la plupart du temps, les interventions de Gaspar et de Raúl le donnent à voir :

25 GAS (...) mucha gente puede morir por la falt- por la falta de $\operatorname{agua}(\ldots)^{20}$

68 RAU (...) la gente que la necesita o sea la va es la que en verdad la necesita $^{21}$

Raúl produit ici une dissociation intéressante sur le plan argumentatif : il précise qu'il parle de ceux qui «ont vraiment besoin» d'eau, en opposition à ceux qui n'en auraient pas «vraiment» besoin. Cela rappelle d'une part que nous avons tous besoin d'eau. D'autre part, cette dissociation insiste sur la situation particulière de certains qui en ont cruellement besoin, c'est-à-dire qui risquent de mourir par manque d'eau.

La gravité de la situation ainsi construite par les élèves impose une certaine "tenue » émotionnelle au cours de la discussion, un devoir moral de «la prendre au sérieux », et facilite l'exploitation des émotions comme ressources pour argumenter.

14 « les riches auront toute l'eau qu'ils veulent et les pauvres non».

15 « elle ne va même pas pouvoir en boire».

16 «les autres gens qui n'ont pas d'eau».

17 « toi tu n'en n'auras pas».

18 « les gens qui n'ont pas d'argent vont se retrouver sans eau peut-être mais peut-être que dans ces endroits où ils vont se retrouver sans eau ».

19 « s'il n'y a pas d'eau économisée comment ils vont à: ces avancées scientifiques sans eau/». 20 « beaucoup de gens peuvent mourir par manq- par manque d'eau ».

21 « les gens qui en ont besoin ou bien ils vont la ce sont ceux qui en ont vraiment besoin ». 


\subsection{L'établissement de deux positions émotionnelles pour deux conclusions opposées}

La discussion tend à se polariser entre deux visions, opposant les partisans de la réponse A (l'accès d'une personne à l'eau potable à l'avenir dépendra surtout de son revenu) et ceux de la réponse C (l'accès d'une personne à l'eau potable à l'avenir dépendra surtout des efforts faits dès maintenant pour économiser l'eau et la préserver). Ces deux affirmations ne sont pas nécessairement opposées. Cependant, le format de l'exercice les oppose puisque les élèves doivent choisir une seule réponse, et que les choix se concentrent entre ces deux options. Ainsi, pendant le débat, les élèves produisent des discours pour défendre l'option qu'ils ont choisie, tout en discréditant l'option « rivale». Ce faisant, ils proposent des descriptions alternatives de la situation, qui orientent fortement vers l'une ou l'autre des conclusions argumentatives en présence. Au travers de ces différences de schématisation (Grize 1996), sont construites deux positions émotionnelles incompatibles, qui soutiennent ces deux conclusions argumentatives.

\section{Construction de la distance à la Question}

Un des éléments structurant la mise en place des émotions sur l'axe de l'intensité correspond, selon Plantin, à la distance que les participants construisent vis-à-vis de leur objet de discours (2011 : 168-171). Ici, le niveau thymique très élevé repose sur une définition du problème comme étant relativement proche des interactants, et ce alors même qu'il s'agit d'élèves de familles aisées qui ne sont pas directement concernés par le manque d'eau. En effet, la distance à la Question n'est pas une donnée, elle se dessine au fil des échanges, selon trois dimensions : qui est concerné ? où et quand?

Le tableau 1 permet de comparer les termes employés par chacun des «camps » pour désigner les personnes concernées par le problème de l'accès à l'eau potable, dans leur ordre d'apparition au fil du discours.

Oana, propose le fait d'avoir ou non de l'argent comme critère de définition des personnes concernées par le problème de l'accès à l'eau potable. Ce même critère est employé par Miguel puis Emilia. Les partisans de la réponse $\mathrm{C}$ contre-argumentent en recadrant le problème comme étant leur problème à tous, avec l'emploi récurrent de la première personne du pluriel. Avec l'intervention de Raúl, émerge un autre critère de définition des personnes concernées : celles qui en ont besoin. Or personne n'échappe à cette grande famille humaine, même si certains en ont plus besoin que d'autres. Il s'agit donc d'un « nous » abstrait. 


\begin{tabular}{|c|c|c|}
\hline Tour $^{22}$ & $\begin{array}{c}\text { Selon les partisans de A } \\
\text { ("de su ingreso económico") }\end{array}$ & $\begin{array}{l}\text { Selon les partisans de C } \\
\text { ("de los esfuerzos") }\end{array}$ \\
\hline $6 \mathrm{OAN}$ & $\begin{array}{l}\text { si tienes dinero obviamente vas a tener } \\
\text { agua (si tu as de l'argent évidemment } \\
\text { tu vas avoir de l'eau) }\end{array}$ & \\
\hline 19 MAR & & $\begin{array}{c}\text { terminemos }(\ldots) \text { para que }(\ldots) \text { no } \\
\text { lleguemos a eso (que nous terminions } \\
(\ldots) \text { pour que }(\ldots) \text { nous n'en arrivions } \\
\text { pas là) }\end{array}$ \\
\hline $38 \mathrm{MIG}$ & $\begin{array}{l}\text { sólo la gente rica va a tener seulement } \\
\text { les gens riches vont en avoir) }\end{array}$ & \\
\hline 42 MAR & & $\begin{array}{l}\text { lleguemos a estos extremos (...) para } \\
\text { que no lleguemos hasta esto } \backslash \text { (que nous } \\
\text { arrivions à ces extrêmités (...) pour que } \\
\text { nous n'en arrivions pas jusque là } \backslash \text { ) }\end{array}$ \\
\hline 46 ART & & $\begin{array}{l}\text { y si hay personas que tienen }(\ldots) \text { más } \\
\text { dinero que tú/ }(\ldots) \text { tú no vas a tener } \backslash \text { (et } \\
\text { s'il y a des personnes qui ont }(\ldots) \text { plus } \\
\text { d'argent que toi }(\ldots) \text { toi tu ne vas pas en } \\
\text { avoir })\end{array}$ \\
\hline 56 GAS & $\begin{array}{c}\text { adaptarme a la situación y buscar } \\
\text { maneras para conseguir dinero } \backslash \\
\text { (m'adapter à la situation et chercher } \\
\text { des manières d'obtenir de l'argent } \ \text { ) }\end{array}$ & \\
\hline $62 \mathrm{OAN}$ & & los pobres (les pauvres) \\
\hline 64 EMI & $\begin{array}{l}\text { la gente que no tiene dinero se va a } \\
\text { quedar sin agua tal vez (...) no } \\
\text { aprovechan bien el agua (les gens qui } \\
\text { n'ont pas d'argent vont se retrouver } \\
\text { sans eau peut-être (...) ils n'exploitent } \\
\text { pas bien l'eau) }\end{array}$ & \\
\hline $66 \mathrm{ADR}$ & & $\begin{array}{l}\text { los que (...) no tienen dinero (ceux qui } \\
\text { n'ont pas d'argent) }\end{array}$ \\
\hline 68 RAÚ & & $\begin{array}{c}\text { la gente que la necesita }(\ldots) \text { que en } \\
\text { verdad la necesita(...) (les gens qui en } \\
\text { ont besoin }(\ldots) \text { qui en ont vraiment } \\
\text { besoin) }\end{array}$ \\
\hline $76 \mathrm{MAR}$ & & $\begin{array}{c}\text { no vamos a llegar a estos extremos } \\
\text { (nous n'allons pas en arriver à ces } \\
\text { extrémités) }\end{array}$ \\
\hline
\end{tabular}

Tableau 1: Personnes concernées par le problème de l'accès à l'eau potable

Arturo, en mettant en scène Gaspar dans un exemple fíctif où il n'aurait pas d'argent, transforme ce "nous» abstrait en un «nous» concret. En se défendant, Gaspar laisse transparaître un autre critère de définition des

${ }^{22}$ Pour l'ensemble des tableaux, la colonne "tour" indique le numéro du tour de parole et les trois premières lettres du locuteur. 
personnes concernées par le problème de l'accès à l'eau : la capacité à «s'adapter» et trouver de l'argent pour subvenir à ses besoins. Cet argument du mérite fait l'objet d'un développement dans une section ultérieure. Cette variation de la distance au problème entre les défenseurs des options $\mathrm{A}$ et $\mathrm{C}$ se construit également sur le plan spatio-temporel.

Emilia, partisane de la réponse A, désigne les lieux concernés avec une forte indétermination, ce qui les présente comme inconnus et lointains :

$64 \mathrm{EMI}(. .$.$) en esos lugares donde se van a quedar sin agua (...) { }^{23}$

Inversement, pour Raúl, tout commence «à la maison », ce qui renvoie à la fois à une proximité spatiale et à une dimension temporelle, en tant que lieu du quotidien :

\section{RAU (...) todo empieza desde la casa $\backslash$ todo $\mid$}

Le problème existe ici selon deux angles différents, qui ne sont ni donnés par la situation, ni construits au hasard. Il n'est neutre ni argumentativement ni émotionnellement de faire entrer le problème à discuter « dans la maison ». La construction de la distance à la question par les partisans de l'option A est susceptible, tout au plus, de produire de la compassion charitable avec les personnes concernées, dans la mesure où elles le méritent. Les partisans de l'option $\mathrm{C}$, au contraire, se présentent comme directement concernés, ce qui est plutôt susceptible de provoquer de la solidarité dans une volonté de se révolter contre une situation injuste, ou au moins de la transformer, soit de l'indignation.

À l'inverse, sur le plan temporel, le camp pro-A a tendance à « réchauffer» émotionnellement le problème, en estimant que le point critique est déjà atteint ou très proche. En effet, la proposition $\mathrm{C}$, suppose qu'il existe un délai minimal nécessaire à l'action avant que les ressources en eau ne soient insuffisantes.

On peut suivre dans le tableau 2 ces deux constructions temporelles du problème. L'entrée en matière de Gaspar présente la situation sous l'angle d'un phénomène spontané, auto-entretenu sans intervention d'un quelconque agent humain (forme pronominale), phénomène qui semble s'accélérer avec l'emploi successif des formes verbales «va commencer à s'épuiser » et « est en train de s'épuiser ». Cet effet de rapprochement suppose qu'entre l'annonce et la réalisation du processus, il n'y a pas de place pour l'action. D'ailleurs, dans la suite du débat, il semble que pour les défenseurs de l'option $\mathrm{A}$, la situation critique soit déjà advenue ou en train d'advenir.

$\mathrm{Au}$ contraire, Maria ou Oana mettent en scène un "nous» comme acteur capable d'intervenir sur la situation problème avant « d'en arriver 
là »: même si le temps presse, il est encore temps d'agir. Raúl, explicite cette construction du problème en insistant sur les effets futurs des comportements actuels. Cette mise à distance "refroidit» la tonalité thymique très négative initiale et offre un espace de contrôle sur le cours des choses.

\begin{tabular}{|c|c|c|}
\hline Tour & $\begin{array}{c}\text { Selon les partisans de A } \\
\text { ("de su ingreso económico") }\end{array}$ & $\begin{array}{l}\text { Selon les partisans de C } \\
\text { ("de los esfuerzos") }\end{array}$ \\
\hline 2 GAS & $\begin{array}{l}\text { se va a empezar a agotar bueno se } \\
\text { está agotando el agua (va } \\
\text { commencer à s'épuiser bon l'eau est } \\
\text { en train de s'épuiser) }\end{array}$ & \\
\hline 19 MAR & & $\begin{array}{l}\text { hasta un momento que (jusqu'à un } \\
\text { moment où) }\end{array}$ \\
\hline 21 EMI & $\begin{array}{l}\text { ya lo estamos agotando (nous } \\
\text { sommes déjà en train de l'épuiser) }\end{array}$ & \\
\hline 42 MAR & & $\begin{array}{l}\text { antes de que lleguemos a estos } \\
\text { extremos (avant que nous n'en } \\
\text { arrivions à ces extrêmités) }\end{array}$ \\
\hline 45 GAS & es la situación (c'est la situation) & \\
\hline 62 OAN & & $\begin{array}{l}\text { para no llegar a estos extremos (pour } \\
\text { ne pas en arriver à ces extrêmités) }\end{array}$ \\
\hline $66 \mathrm{ADR}$ & & después (après) \\
\hline 68 RAÚ & & $\begin{array}{l}\text { lo que pase ahorita }(\ldots) \text { se va a ver } \\
\text { reflejado en las consecuencias } \backslash \text { (ce qui } \\
\text { advient maintenant }(. . .) \text { va se voir } \\
\text { reflété dans les conséquences } \backslash \text { ) }\end{array}$ \\
\hline $76 \mathrm{MAR}$ & & $\begin{array}{c}\text { no vamos a llegar a estos extremos } \\
\text { (nous n'allons pas en arriver à ces } \\
\text { extrémités) }\end{array}$ \\
\hline
\end{tabular}

Tableau 2 : Horizon temporel pour que la situation devienne critique

La construction de la distance temporelle vis-à-vis du problème pose déjà les jalons d'une autre dimension qui oriente émotionnellement et argumentativement le discours : quel degré de contrôle a-t-on sur la situation-problème ? peut-on isoler une cause ou une personne responsable de cette situation?

Différentes constructions de la Question en termes de contrôle possible et de causalitélagentivité

À ces questions, les partisans des réponses $\mathrm{A}$ et $\mathrm{C}$ tendent à répondre différemment. Chaque camp esquisse une représentation adéquate et cohérente pour chacune des deux conclusions argumentatives visées. 
Les interventions concernant le degré de contrôle possible sur l'évolution de la situation sont recensées, dans l'ordre chronologique, dans le tableau 3 ci-après.

\begin{tabular}{|c|c|c|c|}
\hline Tour & $\begin{array}{l}\text { Selon les partisans de A } \\
\text { ("de su ingreso } \\
\text { económico") }\end{array}$ & $\begin{array}{l}\text { Selon les partisans de C } \\
\text { ("de los esfuerzos") }\end{array}$ & $\begin{array}{c}\text { Fonction } \\
\text { argumentative }\end{array}$ \\
\hline 6 OAN & $\begin{array}{c}\text { dices que la ahorras pero } \\
\text { y los que no la ahorran } \\
\text { que pasa (tu dis que tu } \\
\text { l'économises mais et } \\
\text { ceux qui ne } \\
\text { l'économisent pas qu'est- } \\
\text { ce qu'il se passe) }\end{array}$ & & $\begin{array}{l}\text { Opposition à la } \\
\text { proposition C par } \\
\text { passage au } \\
\text { microsocial }\end{array}$ \\
\hline $23 \mathrm{JES}$ & & $\begin{array}{c}\text { si ahorita la cuidamos la } \\
\text { per- la perseveramos }(. . .) \\
\text { no va a tener porque } \\
\text { aumentar (si maintenant } \\
\text { nous y faisons attention, } \\
\text { nous la per- la préservons } \\
\text { (...) il n'y aura pas de } \\
\text { raison que ça augmente) }\end{array}$ & $\begin{array}{l}\text { Réaffirmation } \\
\text { de C }\end{array}$ \\
\hline 25 GAS & $\begin{array}{c}\text { si logra pasarse } \\
\text { ahorramos el agua y sino } \\
\text { (si cela parvient à arriver } \\
\text { que nous économisons } \\
\text { l'eau et sinon) }\end{array}$ & & $\begin{array}{l}\text { Questionnement } \\
\text { de C }\end{array}$ \\
\hline 68 RAÚ & & $\begin{array}{c}\text { lo que pase ahorita }(. . .) \text { se } \\
\text { va a ver reflejado en las } \\
\text { consecuencias } \backslash \text { (ce qui } \\
\text { advient maintenant }(\ldots) \\
\text { va se voir reflété dans les } \\
\text { conséquences } \backslash \text { ) }\end{array}$ & $\begin{array}{l}\text { Réaffirmation de C } \\
\text { et lien avec le } \\
\text { microsocial }\end{array}$ \\
\hline 74 ALE & $\begin{array}{c}\text { aunque la ahorres }(\ldots) \\
\text { alguien más se la va a: se } \\
\text { la va a desgastar (même } \\
\text { si tu l'économises (...) } \\
\text { quelqu'un d'autre va se la } \\
\text { gâcherl) }\end{array}$ & & $\begin{array}{c}\text { Réitération de la } \\
\text { critique microsociale } \\
\text { de C }\end{array}$ \\
\hline $76 \mathrm{MAR}$ & & $\begin{array}{c}\text { si }(\ldots) \text { iniciamos este no } \\
\text { vamos a llegar a estos } \\
\text { extremos (si }(\ldots) \text { nous } \\
\text { commençons euh nous } \\
\text { n'allons pas arriver à ces } \\
\text { extrémités) } \\
\end{array}$ & Réaffirmation de C \\
\hline
\end{tabular}

Tableau 3: Contrôle sur l'évolution de la situation 
Ceux qui pensent que les efforts actuels sont déterminants pour l'accès à l'eau à l'avenir présentent la situation comme pouvant être modifiée grâce à ces efforts. C'est sur ce point que Oana, qui défend alors la position rivale A, exprime un désaccord. À sa suite, Gaspar et Alejandro présentent un tel contrôle sur la situation comme incertain voire impossible. En termes de construction des émotions, penser qu'un contrôle est possible sur l'évolution de l'eau disponible tend à «refroidir» la question, et donc ici à diminuer l'intensité de la tonalité thymique désagréable associée à l'objet du débat. Inversement, soutenir qu'il n'y a pas de contrôle possible sur l'évolution de l'eau disponible produit un sentiment de fatalité qui, associé à la thymique négative initiale, pose la question comme encore plus critique.

Sur le plan argumentatif, ce débat pose déjà une question d'agentivité. Effectivement, c'est par une dissociation que Oana met en cause la proposition $\mathrm{C}$, en distinguant son interlocuteur, volontaire pour préserver l'eau, des personnes qui ne font pas d'efforts. Ainsi, c'est par le déplacement du débat d'un niveau macrosocial à un niveau microsocial qu'est construite la critique à la réponse $\mathrm{C}$. Cette ligne argumentative est suivie par Gaspar, puis Alejandro. Mais aucun partisan de l'option $\mathrm{C}$ ne s'aligne sur cette distinction, et Jesus et Maria réaffirment l'existence d'un "nous» global. Raúl, cependant, offre une variante de ce positionnement «pro-C» qui met l'accent sur la responsabilité individuelle de chacun comme base de l'évolution sociale.

Le degré de contrôle estimé sur la situation est fortement lié à ce qui est considéré comme cause(s) ou agent(s) responsable(s) du problème discuté. Relever ce qui apparaît comme tels dans le discours des deux camps permet de préciser les divergences de leurs positions émotionnelles, et surtout leurs fonctions argumentatives (cf. tableau 4 ci-après).

L'argumentation par la cause/l'agentivité est fortement associée à la construction émotionnelle du débat. En effet, la désignation ou non d'une personne ou d'un phénomène responsable oriente vers la manifestation d'une émotion donnée. Par exemple, la résignation est de mise face à la fatalité, l'indignation face à une injustice flagrante.

Ici, on ne trouve que dans le camp pro-A un cadrage de fatalité, chez Gaspar et Miguel. La formule pronominale de Gaspar, « l'eau est en train de s'épuiser» est focalisé sur l'évolution même de la ressource et donne l'impression d'un phénomène indépendant de l'intervention humaine. Miguel, lui, mobilise l'analogie avec le pétrole : c'est à la fois la fatalité d'une ressource qui est vouée à s'épuiser, et d'une organisation de la société qui ne garantit l'accès aux biens rares qu'aux riches. Cependant, l'emploi de la norme de mérite par certains partisans de $\mathrm{A}$ les amène à formuler tout de même plusieurs formes de responsabilité dans l'évolution de l'accès à l'eau. 
Gaspar invoque ainsi la responsabilité individuelle de s'adapter en trouvant les moyens de subvenir à ses besoins. Les personnes qui n'exploiteraient pas correctement les ressources en eau sont également explicitement mentionnées comme responsables par Oana, Gaspar et Emilia. Ceci n'est pas en contradiction avec le sentiment de fatalité construit parallèlement : la fatalité vient justement du fait qu'il y aura toujours certaines personnes qui ne feront pas les efforts dont parlent les partisans de la réponse C.

\begin{tabular}{|c|c|c|c|}
\hline Tour & $\begin{array}{c}\text { Partisans de A } \\
\text { ("de su ingreso económico") }\end{array}$ & Tour & $\begin{array}{c}\text { Partisans de C } \\
\text { ("de los esfuerzos") }\end{array}$ \\
\hline 2 GAS & $\begin{array}{l}\text { se va a empezar a agotar bueno } \\
\text { se está agotando el agua (va } \\
\text { commencer à s'épuiser bon l'eau } \\
\text { est en train de s'épuiser) }\end{array}$ & \multirow[t]{2}{*}{$23 \mathrm{JES}$} & \multirow[t]{2}{*}{$\begin{array}{c}\text { si ahorita la cuidamos la per- la } \\
\text { perseveramos (si maintenant } \\
\text { nous y faisons attention, la per- } \\
\text { la préservons) }\end{array}$} \\
\hline $6 \mathrm{OAN}$ & $\begin{array}{l}\text { los que no la ahorran (ceux qui } \\
\text { ne l'économisent pas) }\end{array}$ & & \\
\hline $21 \mathrm{EMI}$ & $\begin{array}{l}\text { lo estamos agotando }(\ldots) \text { las } \\
\text { personas (nous sommes en train } \\
\text { de l'épuiser }(\ldots) \text { les personnes) }\end{array}$ & \multirow[t]{2}{*}{68 RAÚ } & \multirow{2}{*}{$\begin{array}{l}\text { gente que tenga mucho dinero y } \\
\text { la tenga pero nada más la va a } \\
\text { desperdiciar (des gens qui ont } \\
\text { beaucoup d'argent et qu'ils l'ont } \\
\text { mais qui ne vont rien faire } \\
\text { d'autre que la gaspiller) }\end{array}$} \\
\hline 25 GAS & $\begin{array}{c}\text { hay varias gentes que no } \\
\text { ahorran (il y a diverses } \\
\text { personnes qui n'économisent } \\
\text { pas) }\end{array}$ & & \\
\hline $38 \mathrm{MIG}$ & $\begin{array}{l}\text { como cualquier recurso como el } \\
\text { petroleo (...) sólo la gente rica } \\
\text { va a tener más disponibilidad al } \\
\text { agua } \backslash \text { (comme n'importe quelle } \\
\text { ressource comme le pétrole }(. . .) \\
\text { seulement les gens riches sont } \\
\text { avoir une plus grande } \\
\text { disponibilité à l'eau })\end{array}$ & 68 RAÚ & $\begin{array}{c}\text { todo empieza desde la casa } \backslash \\
\text { todo } \backslash \text { (tout commence depuis la } \\
\text { maison } \backslash \text { tout } \backslash \text { ) }\end{array}$ \\
\hline 56 GAS & $\begin{array}{l}\text { tendría que buscar (...) o sea no } \\
\text { tener que cambiar el punto de } \\
\text { vista sino tener que adaptarme a } \\
\text { la situación (je devrais chercher } \\
\text { (...) soit non pas avoir à } \\
\text { changer de point de vue mais } \\
\text { avoir à m'adapter à la situation) }\end{array}$ & $76 \mathrm{MAR}$ & $\begin{array}{c}\text { si (...) iniciamos este no vamos } \\
\text { a llegar a estos extremos (si } \\
\text { (...) nous commençons euh } \\
\text { nous n'allons pas en arriver à } \\
\text { ces extrémités) }\end{array}$ \\
\hline 64 EMI & $\begin{array}{l}\text { tal vez (...) no aprovechan bien } \\
\text { el agua y por eso mismo es que } \\
\text { ya: ya es escasa } \backslash \text { (peut-être }(. . .) \\
\text { ils n'exploitent pas bien l'eau et } \\
\text { c'est pour cela même qu'elle est } \\
\text { déjà: déjà rare } \backslash \text { ) } \\
\end{array}$ & & \\
\hline
\end{tabular}

Tableau 4: Agentivité, causalité de l'évolution de l'accès à l'eau 
Dans le camp pro-C, la responsabilité de ceux qui n'exploitent pas correctement l'eau est reconnue également, mais est à la fois étendue à l'échelle de la société, et ramenée au niveau de l'individu. Ce qui compte, c'est l'action de tous, qui commence par le comportement de chacun. L'emploi récurrent de la première personne du pluriel renvoie ainsi à la fois à la société entière, et aux élèves présents dans la salle.

Considérer le «nous », plutôt comme responsable de l'épuisement de l'eau (Emilia) ou plutôt comme capable de l'enrayer (Jesus, Maria) n'est pas argumentativement neutre. De même, évoquer la responsabilité des pauvres incapables d'exploiter l'eau correctement (Emilia) ou rappeler que certains riches gaspillent l'eau (Raúl) n'oriente pas vers le même usage argumentatif de l'idée de mérite. L'analyse de la causalité/agentivité au sein des deux argumentaires laisse apparaître en filigrane deux visions de l'être humain et de la société qui sont à la fois émotionnellement marquées et axiologiquement fondées.

\section{Des normes pour définir les émotions appropriées}

La définition de la causalité/agentivité affecte également la nature plus ou moins agréable des émotions (résignation versus vengeance, par exemple). La conformité aux normes présentées comme étant à suivre participe aussi de la construction du problème en terme d'agrément/désagrément (Ungerer 1997 ; Plantin 2011 : 170).

Le désaccord opposant les camps pro-A et pro-C porte sur l'attitude souhaitable face à une situation-problème grave (cf. 3.1.). Pour faire prévaloir leur réponse sur celle du camp adverse, les interactants ont recours à plusieurs types de normes. Il est utile de distinguer a) les normes fondamentales (notées f), qui correspondent à une certaine vision du monde (valeurs, croyances) ; b) les normes-régularités (notées r), concernant des phénomènes présentés comme des faits non polémiques, et c) les normes de procédure, concernant les règles du jeu du débat, qu'il s'agisse de conditions de pertinence ou de validité des arguments, ou de règles propres à l'exercice (notées p). Une norme fondamentale est rarement explicitée dans sa version générique. Elle peut transparaître dans des jugements évaluant les normesrégularités, ou via les normes de procédure employées.

On peut ainsi suivre comment se co-construisent et se répondent les usages de différentes normes par les deux camps, afin de présenter comme plus ou moins souhaitable la proposition A (tableaux 5 et 6) ou la proposition $\mathrm{C}$ (tableaux 5 bis et 6 bis ci-dessous).

À partir des données, et suivant l'ordre du déroulement réel, sont reconstituées les normes-régularités ( $\mathrm{r} 1, \mathrm{r} 2)$, dont l'évaluation laisse transparaître des normes fondamentales (f1, f2). D'autres normes 
fondamentales sont convoquées indépendamment des normes-régularités (f3, f4 et f5). Des normes de procédure (p1, p2, p3) sont également mobilisées. Lorsqu'une norme est utilisée pour contre-argumenter un argument reposant sur une autre norme, elle est notée avec le même numéro suivi de ', et " lorsqu'il s'agit d'une réponse à une réponse. Lorsqu'un locuteur défendant la même réponse réutilise une norme introduite précédemment, elle est numérotée de la même façon, avec un + , signifiant, le cas échéant, un effet de gradation. Les couleurs permettent de visualiser l'orientation argumentative de cet emploi des normes : gris clair lorsqu'il s'agit de rendre agréable la proposition discutée en la présentant comme conforme aux normes, gris foncé quand il s'agit au contraire d'insister sur sa non-conformité aux normes.

\begin{tabular}{|c|c|c|c|}
\hline Tour & Extrait $^{24}$ & Usage des normes & Effets argumentatifs \\
\hline $6 \mathrm{OAN}$ & $\begin{array}{c}\text { si tu as de l'argent } \\
\text { évidemment tu vas avoir } \\
\text { de l'eau }\end{array}$ & $\mathrm{r} 1: \operatorname{argent}>$ eau & $\begin{array}{c}\text { A proposé comme cause } \\
\text { évidente }\end{array}$ \\
\hline 21 EMI & $\begin{array}{l}\text { il n'y a pas d'autres façons } \\
\text { de raisonner les gens }\end{array}$ & $\mathrm{fl}$ ': $\mathrm{r} 1=$ efficace & $\begin{array}{l}\text { contre-proposition d'un } \\
\text { cadrage émotionnel neutre } \\
\text { pour A }\end{array}$ \\
\hline 45 GAS & $\begin{array}{l}\text { ce n'est pas voir le côté } \\
\text { négatif mais }(\ldots) \text { le } \\
\text { réalisme }(\ldots) \text { ce que moi } \\
\text { j'ai pris en compte c'est la } \\
\text { situation }\end{array}$ & $\begin{array}{l}\mathrm{f} 2^{\prime}: \text { dire }(\mathrm{r} 1)= \\
\text { réaliste ; p1: il faut } \\
\text { argumenter sur le } \\
\text { probable plutôt que } \\
\text { le souhaitable }\end{array}$ & $\begin{array}{l}\text { Refus du cadrage et du } \\
\text { transfert avec la dissociation } \\
\text { yo/tú et tomar en cuenta/ver }\end{array}$ \\
\hline 56 GAS & $\begin{array}{l}\text { j'aurais à chercher }(\ldots) \\
\text { m'adapter à la situation } \\
(\ldots) \text { obtenir de l'argent } \backslash\end{array}$ & $\begin{array}{l}\text { p2': raisonnement } \\
\text { universalisable } \\
\text { f3: mérite }\end{array}$ & $\begin{array}{l}\text { Contre-argumentation par la } \\
\text { cause, le mérite précède la } \\
\text { richesse }\end{array}$ \\
\hline 64 EMI & $\begin{array}{c}\text { peut-être }(\ldots) \text { qu'ils } \\
\text { n'exploitent pas bien l'eau } \\
\text { et pour cela même elle est } \\
\text { déjà: déjà rare }\end{array}$ & f3: mérite & $\begin{array}{l}\text { Contre-argumentation et } \\
\text { légitimation de l'inégalité } \\
\text { sociale par le mérite }\end{array}$ \\
\hline
\end{tabular}

Tableau 5 : La proposition "l'accès à l'eau potable dépendra du revenu" présentée par ses partisans comme conforme aux normes.

Lorsque leur proposition est présentée comme non-conforme à une norme, les élèves réfutent la tonalité négative qu'on tente ainsi de lui associer, et ce par deux moyens. Une première stratégie consiste à chercher à montrer en quoi la proposition en question est en réalité conforme à la norme invoquée. Une autre stratégie correspond au fait de discuter de la pertinence de la norme invoquée, souvent en en invoquant une autre,

${ }^{24}$ Pour des questions de place, nous ne donnons que la traduction des extraits des tableaux 5 et 6. Bien entendu, la version originale est disponible sur demande auprès des auteurs. 
présentée comme supérieure. Dans le premier cas, on se situe au niveau du discours, dans le second, au niveau du métadiscours.

Ainsi, certaines normes invoquées par des élèves ne semblent pas partagées par ceux du camp opposé, qui se réfèrent à d'autres types de normes. On voit nettement que les partisans de la réponse C (l'accès à l'eau dépendra des efforts faits) introduisent volontiers des normes fondamentales (4/5 sur les deux tableaux), alors que ceux qui défendent l'option A (l'accès à l'eau dépendra du revenu) cherchent davantage à discuter des aspects concrets.

\begin{tabular}{|c|c|c|c|}
\hline Tour & Extrait & Usage des normes & Effets argumentatifs \\
\hline 17 MAR & $\begin{array}{l}\text { pourquoi prévaut un } \\
\text { point de vue si triste }\end{array}$ & $\mathrm{fl}:(\mathrm{r} 1)=$ triste & $\begin{array}{l}\text { Opposition basée sur la } \\
\text { tonalité négative de } \mathrm{A}\end{array}$ \\
\hline 42 MAR & $\begin{array}{l}\text { pourquoi toujours voir } \\
\text { du côté négatif }\end{array}$ & f2: dire $(r 1)=$ pessimiste & $\begin{array}{c}\text { Refus du cadrage } \\
\text { "neutre" de A, transfert } \\
\text { de tonalité négative sur } \\
\text { celui choisissant A }\end{array}$ \\
\hline 46 ART & $\begin{array}{l}\text { et s'il y a des personnes } \\
\text { qui ont }(\ldots) \text { plus } \\
\text { d'argent que toi/ }\end{array}$ & $\begin{array}{l}\text { p2 : raisonnement } \\
\text { universalisable }\end{array}$ & $\begin{array}{l}\text { Nouvelle opposition à } \mathrm{A} \\
\text { avec mise en cause de la } \\
\text { personne : ne réfléchit } \\
\text { pas au-delà de sa } \\
\text { position sociale }\end{array}$ \\
\hline 53 RAÚ & $\begin{array}{l}\text { et toi }(\ldots) \text { tu vas le voir } \\
\text { d'un autre point de vue }\end{array}$ & & \\
\hline $62 \mathrm{OAN}$ & $\begin{array}{l}\text { oui cela pourrait être } \\
\text { triste }(\ldots) \text { finir }(\ldots) \text { que } \\
\text { les riches aient toute } \\
\text { l'eau qu'ils veulent et les } \\
\text { pauvres non } \backslash(\ldots) \text { oui tu } \\
\text { as raison } \\
\end{array}$ & $\begin{array}{c}\text { f1: } r 1=\text { triste } \\
\text { f3': égalité des droits } \\
\text { plutôt que mérite ; } \\
\text { p1'+ : le souhaitable } \\
\text { compte, l'émotion } \\
\text { justifie un avis }\end{array}$ & $\begin{array}{c}\text { Opposition à A } \\
\text { réaffirmée, justification } \\
\text { de ce qui est "triste" et } \\
\text { non-souhaitable : } \\
\text { l'inégalité sociale }\end{array}$ \\
\hline 66 ADRI & $\begin{array}{c}\text { ceux qui l'ont } \\
\text { économisée et qui n'ont } \\
\text { pas d'argent }\end{array}$ & \multirow{3}{*}{ f3":mérite } & \multirow{3}{*}{$\begin{array}{c}\text { Retournement de } \\
\text { l'argument du mérite : } \\
\text { les inégalités de richesse } \\
\text { ne sont pas basées } \\
\text { dessus }\end{array}$} \\
\hline 68 RAÚ & $\begin{array}{l}\text { des gens qui ont } \\
\text { beaucoup d'argent et qui } \\
\text { l'ont mais ne vont rien } \\
\text { faire que la gaspiller }\end{array}$ & & \\
\hline 76 MAR & $\begin{array}{l}\text { une personne qui y fait } \\
\text { attention }(. . .) \text { ni elle ne } \\
\text { va pouvoir en boire }\end{array}$ & & \\
\hline
\end{tabular}

Tableau 5 bis: La proposition "l'accès à l'eau potable dépendra du revenu" présentée par les partisans de C ("de los esfuerzos") comme non conforme aux normes.

Par exemple, Oana présente la proposition A comme une normerégularité indiscutable, et ce alors même que les élèves sont dans une phase de la séquence pédagogique clairement intitulée "question d'opinion", donc sans « bonne réponse ». De même, Alejandro, après l'introduction dans 
le débat des normes fondamentales du devoir d'agir sur le monde (f4) et du droit à la satisfaction des besoins vitaux (f5), ne s'oppose à la proposition $\mathrm{C}$ que sur un plan purement technique : la difficulté de conserver l'eau économisée (r2).

\begin{tabular}{|c|c|c|c|}
\hline Tour & Extrait & Usage des normes & Effets argumentatifs \\
\hline 45 GAS & ce que moi j'ai pris en & f2': dire $(\mathrm{r} 1)=$ réaliste \\
& fompte c'est la situation ne peut pas \\
& & $\begin{array}{c}\text { Oppose A à C comme } \\
\text { changer les choses } \\
\text { p1: il faut argumenter } \\
\text { sur le probable plutôt } \\
\text { que le souhaitable }\end{array}$ & \\
\hline 74 ALE & $\begin{array}{c}\text { même si tu l'économise } \\
\text { par exemple tu devrais } \\
\text { la garder toi quelque } \\
\text { part non }\end{array}$ & $\begin{array}{c}\text { p3: importance du } \\
\text { concret }\end{array}$ & $\begin{array}{c}\text { Opposition à C par une } \\
\text { limite technique, refus } \\
\text { de se positionner au plan } \\
\text { moral }\end{array}$ \\
\hline
\end{tabular}

Tableau 6 : La proposition "l'accès à l'eau potable dépendra des efforts faits" présentée par les partisans de A ("de su ingreso económico") comme non conforme aux normes.

\begin{tabular}{|c|c|c|c|}
\hline Tour & Extrait & Usage des normes & Effets argumentatifs \\
\hline 19 MAR & $\begin{array}{l}\text { pourquoi ne pas }(\ldots) \\
\text { commencer à économiser } \\
\text { et pour que }(\ldots) \text { nous n'en } \\
\text { arrivions pas là }\end{array}$ & $\begin{array}{c}\mathrm{f} 1+: \mathrm{r}=\mathrm{a} \text { éviter } \\
\mathrm{f} 4: \text { on peut agir pour cela }\end{array}$ & $\begin{array}{c}\text { Proposition C présentée } \\
\text { comme une alternative } \\
\text { à A }\end{array}$ \\
\hline 42 MAR & $\begin{array}{c}\text { pourquoi }(\ldots) \text { nous ne } \\
\text { commençons pas }(\ldots) \text { il } \\
\text { faut commencer }(\ldots) \text { pour } \\
\text { que nous n'en arrivions } \\
\text { pas jusque là }\end{array}$ & $\begin{array}{c}\mathrm{f} 1+: \mathrm{r} 1 \text { = à éviter } \\
\mathrm{f} 4+: \text { on doit agir pour cela }\end{array}$ & $\begin{array}{c}\text { Proposition C réitérée } \\
\text { comme un devoir d'éviter } \\
\text { A pour l'avenir }\end{array}$ \\
\hline $62 \mathrm{OAN}$ & $\begin{array}{l}\text { oui tu as raison il faut } \\
\text { économiser l'eau pour ne } \\
\text { pas en arriver à ces } \\
\text { extrémités }\end{array}$ & $\begin{array}{c}\mathrm{f} 1+: \mathrm{r} 1 \text { = à éviter } \\
\mathrm{f} 4+: \text { on doit agir pour cela }\end{array}$ & $\begin{array}{c}\text { Réitère } \mathrm{C} \text { comme un } \\
\text { devoir d'éviter } \mathrm{A}\end{array}$ \\
\hline 68 RAÚ & $\begin{array}{l}\text { les gens qui en ont besoin } \\
(\ldots) \text { qui en ont vraiment } \\
\text { besoin }\end{array}$ & $\begin{array}{c}\mathrm{f} 5: \text { droit de tous à la } \\
\text { satisfaction des besoins } \\
\text { vitaux }\end{array}$ & $\begin{array}{l}\text { Justification de C : } \\
\text { respect des besoins vitaux }\end{array}$ \\
\hline
\end{tabular}

Tableau 6 bis : La proposition, "l'accès à l'eau potable dépendra des efforts faits" présentée par ses partisans de $\mathrm{C}$ comme conforme aux normes.

L'intervention de Gaspar, au tour 45, incarne bien ce désaccord entre les deux camps sur une norme de procédure essentielle, qui imprègne l'ensemble de la discussion : peut-on argumenter sur le souhaitable ou doit- 
on s'en tenir au probable (p1)? Alors que Maria, au tour 42, associe la réponse $\mathrm{A}$ et ses partisans à une tonalité émotionnelle négative, Gaspar défend son ethos en effectuant une dissociation entre «tomar en cuenta » (prendre en compte) et "ver» (voir), emblématique de cette opposition souhaitable/probable : « lo que yo tomé en cuenta es la situación». Lui ne ferait que prendre en compte une régularité, et n'aurait en aucun cas le choix de voir les choses sous un aspect ou un autre. Or la manifestation d'un désaccord sur cette règle de procédure n'est évidemment pas un simple différend concernant la forme. Elle va de pair avec le désaccord sur la norme fondamentale 4 concernant la possibilité ou non d'agir sur le cours des choses, et renvoie à la question du contrôle analysée en 3.2.2. Il est à noter que le positionnement par rapport à cette norme $\mathrm{f} 4 \mathrm{n}$ 'a pas seulement pour effet un jugement particulier sur les objets du débat mais également une incidence sur la façon d'argumenter, engageant plutôt à recourir à des normes fondamentales ou à des régularités.

Néanmoins, il existe bien un accord entre l'ensemble des élèves sur plusieurs normes introduites et directement reconnues. D'ailleurs, c'est sans doute l'existence-même de certaines normes faisant autorité pour tous les participants qui permet qu'ait lieu une telle discussion. Lorsque de telles normes sont mobilisées pour rejeter une proposition, la contreargumentation travaille alors davantage à présenter ladite proposition sous un nouveau jour, faisant apparaître sa conformité à la norme partagée. Ainsi, tous semblent adhérer à la norme procédurale selon laquelle un raisonnement n'est acceptable que s'il est généralisable, et non limité aux intérêts de celui qui le produit (p2). Cette norme est convoquée dans une coconstruction entre Arturo et Raúl, et Gaspar cherche à montrer en quoi il la respecte plutôt qu'à questionner cette norme sur un plan métadiscursif.

Quand, au même tour, Gaspar fait entrer dans le débat la norme fondamentale du mérite (f3), les deux stratégies de réponse sont successivement employées par les partisans de C. Oana, au tour 62, moment-clef où elle passe du camp pro-A au camp pro-C, y oppose une autre norme fondamentale : l'égalité de tous les humains. Emilia soutient alors l'argument de Gaspar en établissant un lien entre ceux qui exploitent correctement l'eau et ceux qui y ont accès, fondant une certaine acceptation de l'inégalité sur le mérite. S'ensuit une autre stratégie de réponse de la part d'Adriana, Raul et Maria : la reconnaissance de la norme de mérite et son retournement, en la présentant comme allant à l'encontre de l'option A (l'accès à l'eau dépendra du revenu). Raúl, au tour 68, reprend ces deux éléments : il rappelle d'un côté que le mérite n'est pas nécessairement du côté des riches, et insiste sur l'importance de considérer les besoins vitaux, que chacun a le droit de voir satisfaits (f5). 


\section{L'argument de la tristesse : du conflit de normes au conflit d'émotions}

Une occurrence d'accord sur une norme procédurale mérite tout particulièrement d'être soulignée: le fait que la pertinence d'une argumentation par l'émotion ne soit pas directement contestée. En effet, l'option A est explicitement qualifiée de «triste » à plusieurs reprises, dès le tour 17 par Maria, et cet «argument de la tristesse » est même utilisé par Oana pour motiver son changement d'avis et passer au camp pro-C :

62 OAN (...) sí podría ser triste (...) los ricos tendrán el agua que quieran y los pobres nol (...) si tiene razón hay que ahorrar agua para no llegar a estos extremos (... $)^{25}$

Si cette norme procédurale n'est jamais thématisée, tout au long du débat, les partisans de l'option A travaillent à faire accepter une autre schématisation de leur proposition, pour la défaire de cette tonalité négative. C'est un conflit d'émotions qui se joue dans la qualification du choix de la réponse A comme pessimiste/déprimés/résignés ou comme « réalistes ».

\section{Conclusion}

$\mathrm{Au}$ cours de cet exercice, les élèves, face à une question à choix multiple, débattent en proposant un cadrage de la situation-problème qui restreint le champ des possibles, et leur permet de se positionner et de soutenir leur position. Une dimension importante de ce cadrage est émotionnelle. La position émotionnelle construite oriente effectivement vers la conclusion argumentative correspondante. Ainsi, l'analyse comparée de la construction émotionnelle du problème de l'accès à l'eau potable par les différents «camps», permet de retracer une image précise de la vision proposée par chaque camp au fil de différents " paramètres émotionnants ». Par exemple, l'emploi du pronom «nous » par le camp pro-C participe de la schématisation émotionnelle à différents niveaux. Il renvoie tantôt à une proximité par identification aux personnes concernées, qu'elle soit concrète (groupe-classe) ou abstraite (au niveau de la société); tantôt à la mise en scène d'une instance de contrôle possible ; tantôt à l'introduction d'un agent responsable causalement de l'état de la situation. À l'opposé, le camp pro-A montre une moindre identification aux personnes concernées, et tend plutôt à cadrer le problème comme un phénomène indépendant de l'intervention humaine et autoentretenu, sur lequel on ne pourrait avoir prise.

25 oui, ça pourrait être triste (...) que les riches aient toute l'eau qu'ils veulent et les pauvres non $\backslash(\ldots)$ oui elle a raison il faut économiser l'eau pour ne pas en arriver à ces extrémités de: (...) 
Cette focalisation sur l'émotionnel ne doit pas faire perdre de vue que la représentation émotionnelle du problème ainsi construite fait partie intégrante de sa schématisation qui, toute entière, "oriente" le discours vers une conclusion argumentative. Ce n'est donc finalement que mise en relation avec la conclusion argumentative qui en découle (ici les réponses A ou C) que cette construction émotionnelle prend tout son «sens» argumentatif. Les options d'interprétation du problème ont des conséquences directes sur les façons possibles d'y répondre. Si «dans la parole commune, construire une conclusion argumentative, c'est construire une position émotionnelle » (Plantin 2011:5), il semble que, dans cette situation argumentative spécifique, construire une position émotionnelle, c'est choisir une conclusion argumentative.

Un niveau thymique très intense semble partagé par tous les participants, qui cadre le problème de l'accès à l'eau potable négativement, comme impliquant un risque sérieux d'un manque d'eau mortel. Cependant, les participants jouent sur la «tonalité émotionnelle» des différentes propositions pour les rendre plus ou moins acceptables, et argumenter en faveur de l'une ou de l'autre. La proposition $\mathrm{A}$ se voit très tôt attribuer une tonalité émotionnelle négative (tour 17) qui semble déterminante dans la conversion d'Oana au camp pro-C, au tour 62. Les partisans de la position A n'expriment pas un désaccord explicite sur le fait de mobiliser ainsi les émotions pour argumenter. Au contraire, ils se soumettent à cette norme procédurale et entrent dans le jeu de la (dé)construction émotionnelle de leur proposition en travaillant activement à la montrer comme neutre émotionnellement. L'enjeu est également de réparer leur ethos qui est également négativement marqué par cette tonalité émotionnelle hétéroattribuée.

Suivre la construction argumentative des émotions dans le discours des élèves se révèle un précieux outil méthodologique. Ce type d'analyse fournit un véritable éclairage pour comprendre ce type de débats. Pour la recherche sur le fonctionnement des émotions en argumentation, cette étude de cas fournit un bon exemple de l'impossibilité, dans les discours authentiques, de séparer émotion et raison. La mobilisation des émotions participe de la cohérence du cadrage argumentatif du problème et se fonde sur des normes, des savoirs et des savoir-faire soit explicitement justifiés soit culturellement acceptées comme «raisonnables». Des recherches futures sont néanmoins nécessaires pour mieux comprendre cette l'articulation de l'axiologique (normes fondamentales) et de l'émotionnel. 


\section{Références bibliographiques}

ALBE, Virginie (2006), «Procédés discursifs et rôles sociaux d'élèves en groupes de discussion sur une controverse socio-scientifique». Revue française de pédagogie, 157, 103-118.

CAFFI, Claudia \& JANNEY, Richard (1994), «Toward a pragmatics of emotive communication ». Journal of pragmatics, 22 (3-4), 325-373.

FOWLER, Samantha, ZEIDLER, Dana \& SADLER, Troy (2009), «Moral sensitivity in the context of socioscientific issues in high school science students ». International Journal of Science Education, 31(2), 279-296.

GRIZE, Jean-Blaise (1996), Logique naturelle et communications. Paris: Presses universitaires de France.

HAMBLIN, Charles (1970). Fallacies (Vol. 439). Methuen.

KACEM, Saida, \& SIMONNEAUX, Laurence (2009), "The teaching of socioscientific issues in interdisciplinarity biology-philosophy, an ethical stake and citizenship issue ». US-China Education Review, 6(2), 44-47.

MICHELI, Raphaël (2013). «Esquisse d'une typologie des modes de sémiotisation verbale de l'émotion ». Semen, 35.

MICHELI, Raphaël (2010). L'émotion augmentée: l'abolition de la peine de mort dans le débat parlementaire français. Paris : Cerf.

NUANGCHALERM, Prasart (2010). «Engaging students to perceive nature of science through socioscientific issuesbased instruction $» m$ European Journal of Social Sciences, 13(1), 34-37.

PERELMAN, Chaïm \& Olbrechts-Tyteca, Lucie, (1958), Traité de l'argumentation: la nouvelle rhétorique. Paris : Presses universitaires de France.

PLANTIN, Christian, DOURY, Marianne \& TRAVERSO, Véronique (2000), Les émotions dans les interactions. Lyon : Presses universitaires de Lyon.

PLANTIN, Christian (2011), Les bonnes raisons des émotions - Principes et méthode pour l'étude du discours émotionné. Berne : Peter Lang.

Real Academia de la lengua Española (2001), "agotar", Dictionnaire en ligne, $22^{\text {ème }}$ édition, consulté le 30 octobre 2012 : http://lema.rae.es/drae/?val=agotar.

TOULMIN, Stephen E. (1958), The uses of argument. Cambridge : Cambridge University Press.

UNGERER, Friederich (1997). «Emotions and emotional language in English and German news stories ». The Language of emotions, Amsterdam/Philadelphia: John Benjamins, 307-328.

WALTON, Douglas (1992), The place of emotion in argument. Pennsylvania State University Press. 


\title{
Quand construire une position émotionnelle, c'est choisir une conclusion argumentative : le cas d'un café-débat sur l'eau potable au Mexique
}

Résumé : Cet article propose une étude du travail discursif des participants à un débat pour construire une position émotionnelle cohérente avec la conclusion argumentative qu'ils défendent. Le cas présenté, un café-débat sur l'accès à l'eau potable à l'avenir, mené au sein d'un établissement scolaire mexicain, donne à voir que ce travail porte à la fois sur la tonalité émotionnelle de la question discutée et sur les différentes alternatives de réponse en présence. Les outils méthodologiques proposés par Plantin (2011) sont utilisés pour suivre deux cadrages émotionnels concurrents qui émergent dans le débat, fondés sur une tonalité thymique consensuelle très négative. On analyse quatre paramètres: distance vis-à-vis du problème, causalité/agentivité, possibilité de contrôle et conformité aux normes partagées. La démarche permet de mettre en lumière la pertinence argumentative de la dimension émotionnelle de la schématisation (Grize, 1996) comme processus d'orientation. Dans la dernière section, une attention particulière est portée aux relations entre normes et émotions.

Mots-clés : Schématisation émotionnelle, Interactions entre élèves, Orientation argumentative, Normes, Question socio-scientifique

\section{When to construe an emotional position is to choose an argumentative conclusion : the case of a student's debate on drinking water in Mexico}

\begin{abstract}
This paper consists of a detailed analysis of how the participants to a debate build their emotional position during the interaction and how such a position is related to the conclusion they defend. Our case study of teenage Mexican students arguing on access to drinking water in the future shows extensive discursive work on the emotional tonality given to the issue and to each alternative of response. Methodological tools proposed by Plantin (2011) are used to follow two alternative emotional framings made by opposing groups of students, starting from a common, highly negative, thymical tonality. Through the analysis of four parameters (distance to the problem; causality/agentivity; possibility of control and conformity to the shared norms) we describe how the emotional dimension of the schématisation (Grize, 1996) is argumentatively relevant as an orientation/orientating process. A special focus is made in the last section on the relationship between norms and emotions.
\end{abstract}

Keywords: Emotional schématisation or framing, Student-student interactions, Argumentative orientation, Norms, Socio-scientific issue 